\section{Ford nutzt die Vorteile von Flächendichtungen}

m Ford Fox 1-l-Motor, der nach seiner Einstandardmäßig im Ford Focus sowie zahlreichen zukünftigen Modellen zum Einsatz kommen soll, übernehmen Loctite-Produkte wichtige Abdichtfunktionen. So dient zum Beispiel das bei Raumtemperatur vernetzende Silikonmaterial Loctite 5900 zum Abdichten der Ölwanne sowie der Frontabdeckungsanschlüsse. Diese thixotrope schwarze Paste zeigt nach dem Aushärten eine hervorragende Motorölverträglichkeit und verträgt auch stärkere Vibrationen.

Beim Fox-Motor kommt außerdem der anaerobe, gelförmige Klebstoff Loctite 5182 zum Einsatz, der zur Abdichtung von Nockenwellendeckeln gezielt modifiziert wurde. Der maximale Durchmesser der Dichtmittelraupe ist bei diesem Motor klein. Des-

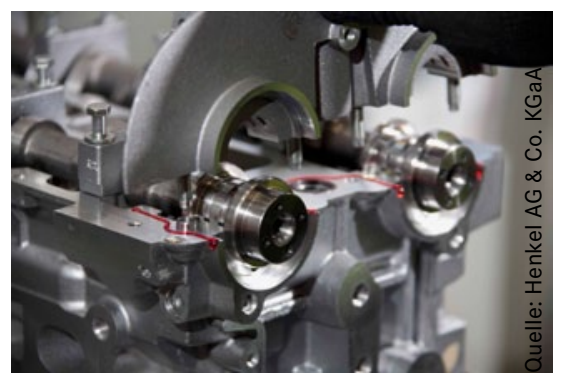

Die Nockenwellendeckelabdichtung wird beim Fox-Motor durch Einsatz eines anaeroben, gelförmigen Klebstoffs realisiert. halb muss das Gel unbedingt exakt und blasenfrei aufgetragen werden, damit die Raupe unterbrechungsfrei verläuft; schon kleine Unregelmäßigkeiten könnten die Motorabdichtung gefährden. Um solche Probleme zu vermeiden, wird das Produkt jetzt während der Herstellung zentrifugiert.

Auch für die Dichtung des Benzinpumpendeckels beim Sigma-Motor wurde der Einsatz dieses anaeroben Klebstoffs in Betracht gezogen. Der neue Ecoboost-Motor wird wie der Ford Fox 1-l-Motor hohen Belastungen ausgesetzt. Allerdings stellte sich bei Tests heraus, dass der größere Ecoboost-Motor einen flexiblen anaeroben Dichtstoff erforderte, sodass Modifizierungen nötig wurden, die in der Entwicklung der anaeroben Flächendichtung Loctite 5188 mündeten.

Bei den erwähnten bei Raumtemperatur härtenden und anaeroben Materialien handelt es sich um selbstformende Dichtungen, die maximalen Flächenkontakt gewährleisten und Korrosion an den abgedichteten Flächen ausschließen. Es bildet sich direkt bei der Montage eine Niederdruckdichtung, die binnen 24 Stunden komplett aushärtet und eine nicht nachgebende, schrumpf- und rissfreie Verbindung schafft. Diese Produkte sind optimal geeignet für die automatische Applikation und erfüllen auch Fords Anforderungen hinsichtlich Zykluszeiten und Reproduzierbarkeit.

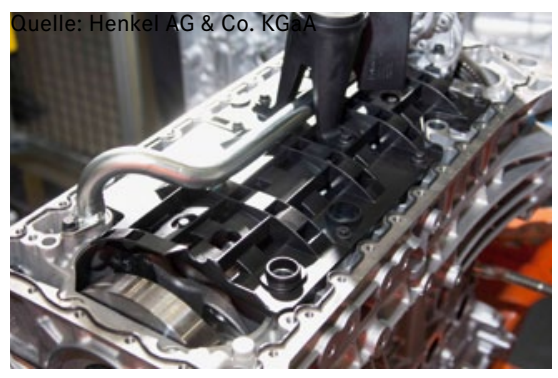

Das für die Abdichtung der Ölwanne verwendete Silikonmaterial vernetzt bei Raumtemperatur, zeigt nach dem Aushärten eine hervorragende Motorölverträglichkeit und verträgt auch stärkere Vibrationen.

Der automatische Auftrag dieser Produkte erfordert zwar ähnliche Kapitalinvestitionen wie der Einsatz von Feststoffdichtungen, ermöglichen aber aufgrund ihrer Flexibilität erhebliche Kosteneinsparungen im laufenden Betrieb. Ein weiterer Vorteil der Flächendichtungen liegt in der Möglichkeit der Abdichtung rauerer und poröserer Oberflächen, sodass bei entsprechenden Toleranzen die Nachbearbeitung entfallen kann. Außerdem erfordern Flächendichtungsmaterialien im Vergleich zu Pressdichtungen deutlich geringere Klemmkräfte, geben nicht nach und halten damit die Vorspannkraft aufrecht. Dies wirkt sich positiv auf die Gewährleistung aus.

Weitere Infos: Henkel AG \& Co. KGaA, www.henkel.de

\title{
1K-Klebstoff zum Kleben von Fördergurten
}

D em Unternehmen ContiTech ist es in Zusammenarbeit mit dem Klebstoffhersteller H.B. Fuller gelungen, einen einkomponentigen Klebstoff zu entwickeln, der den hohen Ansprüchen in der Fördertechnik gerecht wird. In diesem Bereich ist es heute technischer Standard, für bestimmte Arbeiten aus Zeit- und Kostengründen anstelle der früheren Warmvulkanisation die Kaltklebtechnik mit Industrieklebstoffen einzusetzen.

Der neue Klebstoff mit dem Markennamen Conti Secur Premium verspricht im Vergleich zu vorher in diesem Bereich verwendeten Zwei-Komponenten-Klebstoffen mehr Sicherheit trotz einfacherer Verarbeitung. Diese in 0,8-Liter-Gebinden erhältliche Klebstoff auf Basis von Polychloropren ist universell einsetzbar und eignet sich für unterschiedliche Einsatzbereiche. Dazu gehören das Kleben von Gummi mit Gummi und Gummi mit Metall oder auch Verbinden von Gewebegurten.

Ein weiterer Pluspunkt ist das geringere Verpackungsvolumen, da die Verstärkerlösung entfällt. Das reduziert die Kosten sowohl bei Versand und Lagerung als auch bei der Abfallentsorgung.

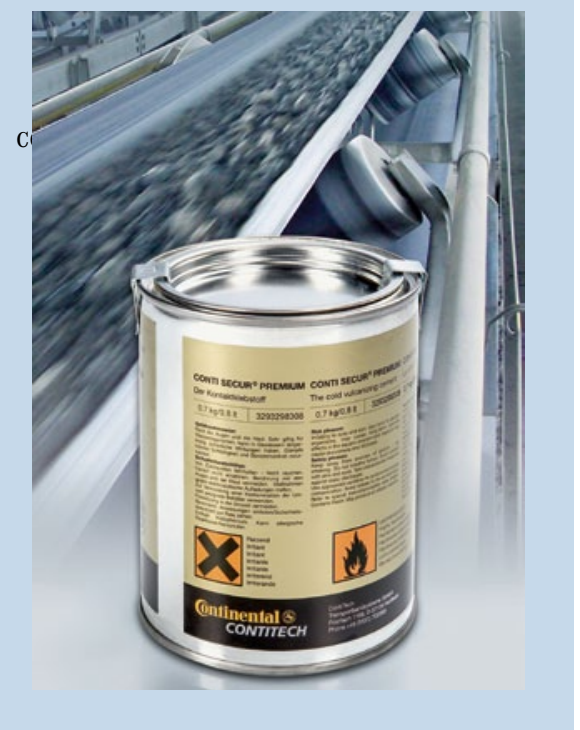

